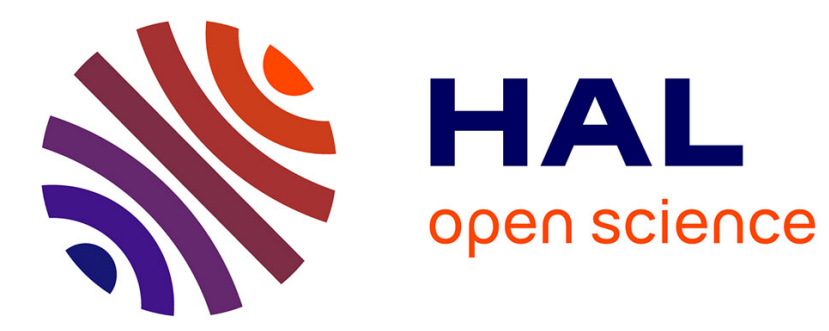

\title{
Experimental characterization of recurrences and separatrix crossing in modulational instability
}

Corentin Naveau, Pascal Szriftgiser, Alexandre Kudlinski, Matteo Conforti, Stefano Trillo, Arnaud Mussot

\section{To cite this version:}

Corentin Naveau, Pascal Szriftgiser, Alexandre Kudlinski, Matteo Conforti, Stefano Trillo, et al.. Experimental characterization of recurrences and separatrix crossing in modulational instability. Optics Letters, 2019, 44 (22), pp.5426. 10.1364/OL.44.005426 • hal-02384605

\section{HAL Id: hal-02384605 \\ https://hal.science/hal-02384605}

Submitted on 28 Nov 2019

HAL is a multi-disciplinary open access archive for the deposit and dissemination of scientific research documents, whether they are published or not. The documents may come from teaching and research institutions in France or abroad, or from public or private research centers.
L'archive ouverte pluridisciplinaire HAL, est destinée au dépôt et à la diffusion de documents scientifiques de niveau recherche, publiés ou non, émanant des établissements d'enseignement et de recherche français ou étrangers, des laboratoires publics ou privés. 


\title{
Experimental characterization of recurrences and separatrix crossing in modulational instability
}

\author{
Corentin naveau, ${ }^{1,}{ }^{*}$ Pascal Szriftgiser, ${ }^{1}$ Alexandre Kudlinski, ${ }^{1}$ Matteo \\ Conforti, ${ }^{1}$ Stefano Trillo, ${ }^{2}$ And Arnaud Mussot ${ }^{1}$ \\ ${ }^{1}$ University of Lille, CNRS, UMR 8523 - PhLAM - Physique des Lasers Atomes et Molécules, Lille, France \\ ${ }^{2}$ Department of Engineering, University of Ferrara, Ferrara, Italy \\ *Corresponding author: corentin.naveau@univ-lille.fr
}

Received XX Month XXXX; revised XX Month, XXXX; accepted XX Month XXXX; posted XX Month XXXX (Doc. ID XXXXX); published XX Month XXXX

\begin{abstract}
We experimentally investigate two cycles of Fermi-PastaUlam-Tsingou recurrence in optical fibers. Using three waves input we characterize the distance of maximum compression points against sideband amplitude and relative phase, outlining the qualitative changes of the dynamics due to separatrix crossing. Experimental results are in good agreement with numerical simulations and analytical predictions.
\end{abstract}

OCIS codes: (190.4370) Nonlinear optics, fibers; (190.4223) Nonlinear wave mixing

Fermi-Pasta-Ulam-Tsingou (FPUT) recurrences correspond to the ability of a multimodal system experiencing complex nonlinear dynamics to come back to its initial state [1]. Beyond this first step, these systems can exhibit multiple recurrence cycles before eventually reaching energy equipartition between the modes $[2$, 3]. Initially studied in the context of oscillator chains, the FPUT recurrence process can also be observed in many different frameworks such as plasma physics [4], hydrodynamics [5, 6] and optics [7-17]. In optics, FPUT has been investigated in particular in the context of modulation instability (MI) triggering the process [7]. MI consists in the exponential growth of a weak periodic modulation at the expense of a strong continuous pump wave [18]. Then, in $\chi^{(3)}$ media such as silica optical fibers, harmonics are generated via subsequent four-wave mixing processes between the waves to form a triangular shaped spectrum [19] until the process reverses and the system returns to its initial state. This process has attracted a tremendous interest as it plays a key role in many nonlinear phenomena governed by the nonlinear Schrödinger equation (NLSE) such as rogue wave formation and supercontinuum generation [20,21]. Until recently, experimental observations were limited to a single growth and decay recurrence cycle $[7,13,14]$. Such dynamics has been described by exploiting the exact solution of the NLSE known as Akhmediev breather (AB) $[20,22]$, which represents a separatrix orbit emanating and returning at infinite distance to the pump wave though with a phase shift. On this basis, assuming three waves as initial conditions, a simple expression predicting the position of the maximum depletion (maximally compressed pulse train) has been derived [23, 24]. However, this expression is limited to a symmetric modulation and does not allow to account for the impact of the initial relative phase between the pump and the modulation signal, which is known to have a strong impact on the dynamics $[9,10]$. Furthermore, more recent experiments in water waves [6], in fiber optics $[15,16]$ or in a bulk photorefractive crystal [17] has shown that two recurrence cycles become accessible.

All this calls for a more insightful theoretical approach that allows to describe the rich dynamics involved in the two recurrence cycles. In principle one could use general doubly periodic solutions of the NLSE developed by Akhmediev et al. [25] but these in general do not reduce to a spectrum with three frequencies thus making difficult to properly fit with the input three waves mostly used in experiments [23]. A significant step forward has been made by Grinevich and Santini [26], who considerably improved the matching theory with the $\mathrm{AB}$, being able to characterize the dependence of the first compression point and the spatial period of successive recurrences on the parameters of three wave input. Grinevich and Santini's predictions have been recently verified experimentally in a spatial MI experiment involving a bulk 3D photorefractive crystal [17]. The aim of this letter is to report the experimental test of such predictions for fiber optics. Fibers indeed constitute one of the most convenient test bed to investigate FPUT process offering easy tuning of initial conditions, good control of fiber parameters and the opportunity of compensating losses while performing non-destructive measurements of the full (modulus and phase) electric field along the whole fiber length $[15,16]$.

The experimental setup is basically the same than in Ref. [15]. We used a $9.2 \mathrm{~km}$ SMF-28 fiber and launched three waves at the input (i.e. a pump, signal and idler). To obtain this input, we generate first a frequency comb with a $35 \mathrm{GHz}$ line spacing via phase modulation of a laser. A commercial programmable optical filter (Waveshaper) allows then to tailor this comb in order to keep 
only three waves and to precisely control their amplitudes and relative phase. To characterize the evolution of these waves, we used then a setup which consists in a heterodyne optical timedomain reflectometer [15]. Through the coherent detection of the Rayleigh backscattered waves, it allows to perform distributed measurements along the fiber of the phase and intensity of the pump, signal and idler waves. The longitudinal resolution of our system is about $20 \mathrm{~m}$. Moreover, our set-up also features active compensation of the intrinsic fiber losses via a counterpropagative Raman amplification scheme. This allows to consider our system as conservative, so that its dynamics can be described by the following integrable NLSE:

$$
i \frac{\partial \Psi}{\partial Z}-\frac{\beta_{2}}{2} \frac{\partial^{2} \Psi}{\partial T^{2}}+\gamma|\Psi|^{2} \Psi=0
$$

where $\Psi(Z, T)$ is the field envelope, $\mathrm{Z}$ and $\mathrm{T}$ account for the distance along the fiber and the retarded time, respectively, $\beta_{2}$ is the group velocity dispersion and $\gamma$ is the nonlinear coefficient. Our experiments are then described by the following initial condition:

$$
\Psi(Z=0, T)=\sqrt{P_{0}}\left(e^{i \Phi_{0}}+c_{1} e^{i \Omega T}+c_{-1} e^{-i \Omega T}\right)
$$

where $P_{0}$ and $\Phi_{0}$ are the pump power and phase, respectively, $\Omega$ the modulation angular frequency, $c_{ \pm 1}=\left|c_{ \pm 1}\right| e^{i \Phi_{1}}$, where $\left|c_{ \pm 1}\right| \ll 1$ are the normalized amplitude of the signal and idler wave, respectively, and $\Phi_{1}$ their phase. Using an equivalent ansatz in Ref. [26], Grinevich and Santini have derived the positions $Z_{1}$ and $Z_{2}$ of the first and second maximum compression points which read respectively as

$$
\begin{gathered}
Z_{1}=\frac{2}{g} \ln \left(\frac{g^{2}}{2|\alpha|}\right) \times L_{N L} \\
Z_{2}=\frac{4}{g} \ln \left(\frac{g^{2}}{2 \sqrt{|\alpha \beta|}}\right) \times L_{N L}+Z_{1}
\end{gathered}
$$

where $g=\omega \sqrt{4-\omega^{2}}$ is the MI gain, with $\omega=\Omega \sqrt{\left|\beta_{2}\right| L_{N L}}$ the normalized modulation angular frequency, $\alpha=c_{1}^{*}-e^{i 2 \Phi_{\omega}} c_{-1} \quad$ and $\quad \beta=c_{-1}^{*}-e^{-i 2 \Phi_{\omega}} c_{1} \quad$ with $\Phi_{\omega}=\arccos \left(\frac{\omega}{2}\right)$ and $L_{N L}=\left(\gamma P_{0}\right)^{-1}$ the nonlinear length.

Using our experimental setup, we are then able to investigate the impact of the initial modulation sideband amplitude and relative phase between the pump and sidebands $\left[\Delta \Phi_{0}=\Phi_{0}(\mathrm{z}=\right.$ $\left.0)-\Phi_{1}(z=0)\right]$ on the two first FPUT recurrence cycles and to compare the positions of these recurrences with the previous estimates from Eqs. (3-4). To do so, we used a $470 \mathrm{~mW}$ pump wave with a $35 \mathrm{GHz}$ modulation and tuned the initial sidebands amplitude and phase via the Waveshaper. An example of experimental recording is showcased in Fig. 1. In this case, the initial sideband powers are equal and their phases identical. The signal/idler to pump ratio was set to $-10.4 \mathrm{~dB}$ and the initial relative phase to $\Delta \Phi_{0}=0.50 \pi$ [see the spectrum of the simulated input in Fig. 1(a)]. The experimental longitudinal evolution of the pump and signal power are then displayed in solid lines in Fig. 1(b). We observe almost two complete conversion cycles, in good agreement with simulations (obtained via the numerical
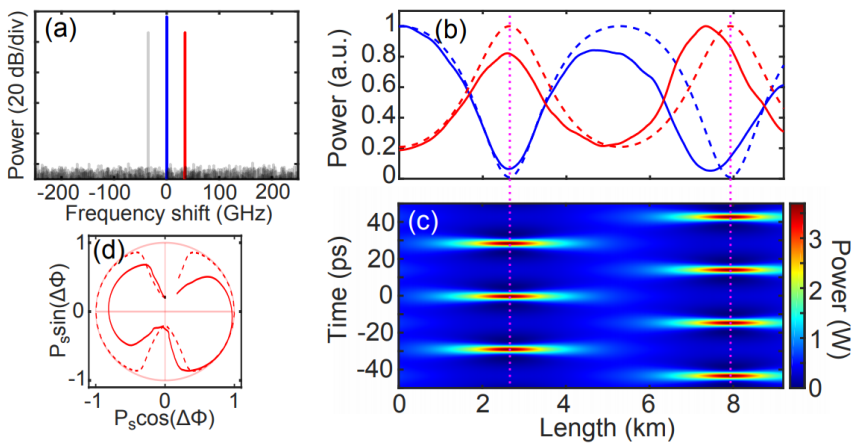

Fig. 1. (a) Simulated input spectrum. (b) Longitudinal evolution of the pump (in blue) and signal (in red) power for $\Delta \Phi_{0}=0.50 \pi$. (c) Simulated spatio-temporal evolution of power. The magenta dashed lines highlight the position of the maximum compression points. (d) Corresponding phase-plane structures. Solid lines correspond to experiments and dashed ones to numerical simulation. All power plots are normalized to their maxima. Parameters: fiber length $\mathrm{L}=9.2 \mathrm{~km}$, group velocity dispersion $\beta_{2}=-19 \times$ $10^{-27} \mathrm{~s}^{2} / \mathrm{m}$, nonlinear coefficient $\gamma=1.3 \times 10^{-3} / \mathrm{W} / \mathrm{m}$, pump power $\mathrm{P}_{0}=470 \mathrm{~mW}$, signal to pump ratio $-10.4 \mathrm{~dB}$, signal detuning $35 \mathrm{GHz}$ from the pump.

integration of the NLSE) displayed in dashed lines. These cycles can also be seen in Fig. 1(c), which illustrates the simulated spatiotemporal evolution of the power. Moreover, the setup allows also to measure the relative phase $\Delta \Phi(z)$ between the pump and the sidebands whose impact is represented by drawing the trajectories in a phase plane $\left(P_{s} \cos \Delta \Phi, P_{s} \sin \Delta \Phi\right), P_{s}(z)$ being the signal power fraction [see Fig. $1(\mathrm{~d})][9,10,15]$. In the case shown, the $\pi$ (half-period) shift in time domain of the second apex conversion (Fig. 1 (c)) is the signature that the mixing follows a figure of eight outer trajectory with respect to the separatrix. Conversely, inner trajectories are characterized by consecutive inphase recurrences and are confined in left or right semi-phaseplane (see discussion below). Noteworthy, the position of the pump minima nearly coincides with the maximum compression points (as highlighted by the magenta dashed lines in Figs 1(b,c)). Hence, in what follows, we will focus on the position of these pump minima to characterize the FPUT process.

First, we investigated how the input relative phase $\Delta \Phi_{0}$ between the pump and two sidebands with equal amplitude affects the recurrence distances, comparing with Eqs. (3-4). To this end, we measure the evolutions (as in Fig. 1) by varying $\Delta \Phi_{0}$ from $-\pi / 2$ to $\pi / 2$ while maintaining the signal/idler to pump power ratio fixed at $-10.4 \mathrm{~dB}$. The results are summarized in Fig. 2(a) which shows the position of the first (green) and second (magenta) pump minima. Crosses account for the experimental recordings, solid lines for the theoretical predictions [Eqs. (3-4)] and dashed lines for numerical simulations. Clearly, the finite fiber length highlighted by the horizontal grey line sets an upper bound to measurable distances. Significantly, Eqs. (3-4) predict that both $Z_{1}$ and $Z_{2}$ diverges for $\Delta \Phi_{0}=\Phi_{\omega}$ (in our case $\Phi_{\omega}=0.29 \pi$ ). This is because such phase corresponds to the stable manifold of the separatrix (or $\mathrm{AB}$ solution) entailing asymptotic conversion from the input sidebands to the pump. Conversely, on the unstable manifold corresponding to $\Delta \Phi_{0}=-\Phi_{\omega}$ the sidebands experience the most rapid growth to the apex, thus yielding the minimum $Z_{1}$, whereas $Z_{2}$ diverges since backconversion still occurs 

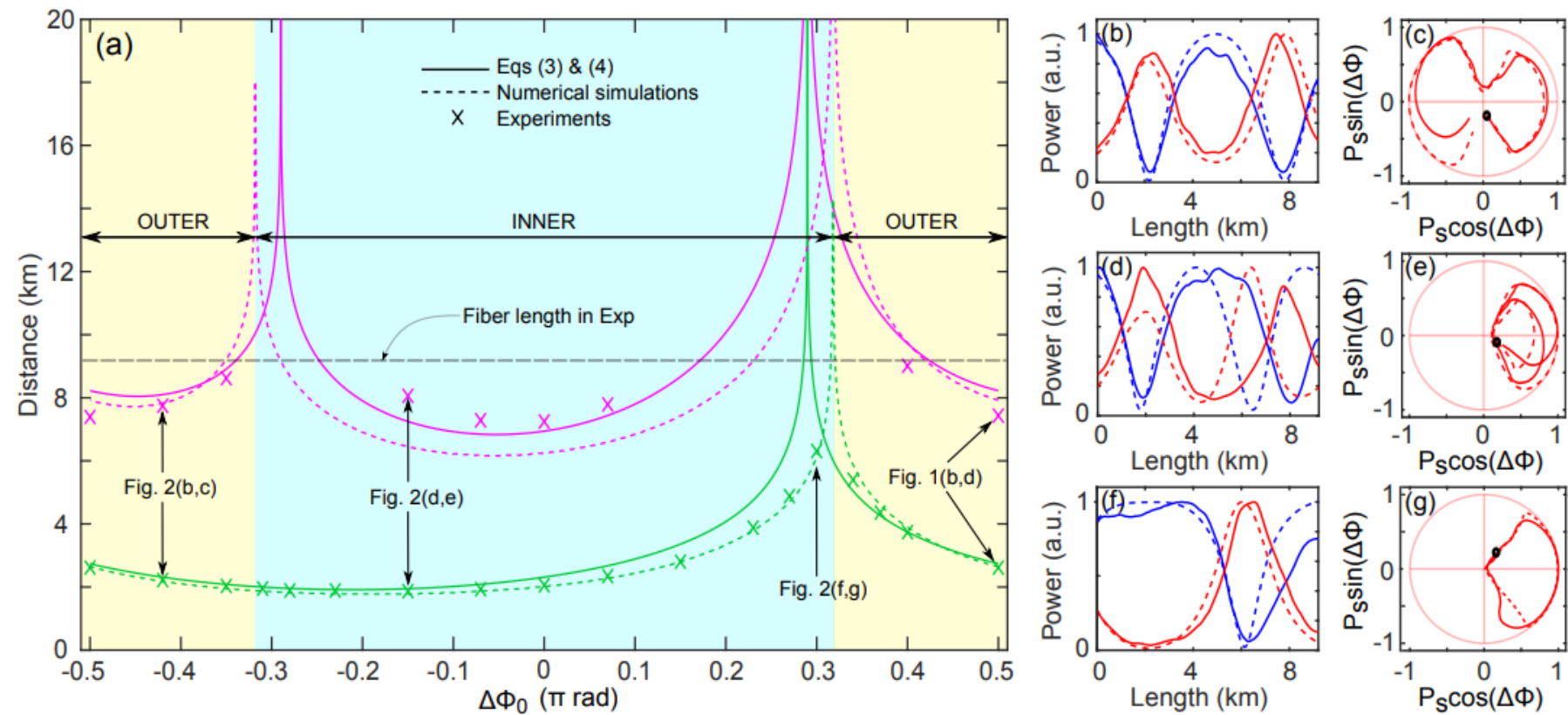

Fig. 2. (a) Positions of the first (green) and second (magenta) pump minima. The crosses correspond to experimental recordings, dashed lines to numerical simulations from the NLSE and solid lines to the theoretical model. (b,d,f) Longitudinal evolution of the pump (blue) and signal power (red) and (c,e,g) their corresponding phase plane trajectories, for $\Delta \Phi_{0}=-0.42 \pi, \Delta \Phi_{0}=-0.15 \pi$ and $\Delta \Phi_{0}=0.30 \pi$, respectively. Solid lines correspond to experiments and dashed ones to numerical simulations. The grey line indicates the length of the fiber. All power plots are normalized to their maxima.

asymptotically along the separatrix. Incidentally, for $\Delta \Phi_{0}=-\Phi_{\omega}$, such minimal $Z_{1}$ from Eq. (3) coincides with the estimate of Ref. [23]. Conversely, it underestimates $Z_{1}$ for any other values of input phase. Importantly, the values $\Delta \Phi_{0}= \pm \Phi_{\omega}$ represent the critical phases (in the limit of very weak sidebands) across which the nonlinear MI undergoes separatrix crossing $[9,10,15]$, or in other words transition from shifted to unshifted recurrences. However, the NLSE simulations as well as the measured value of $Z_{1}$, which are in excellent agreement with the numerics, indicate that this transition occurs at slightly shifted value of input phase $\Delta \Phi_{0}=$ $\pm \Delta \Phi_{c}$ with $\Delta \Phi_{c}=0.32 \pi$ (instead of $0.29 \pi$ ) due to the relatively strong modulation (in our setting the sidebands contain nearly $15 \%$ of total input power). In Fig. 2(a) such values mark the border between yellow and cyan shaded areas, in turn yielding measured evolutions along outer orbits (phase-shifted recurrences) and inner orbits (unshifted recurrences), respectively. Examples of outer orbits yielding alternate shifted recurrences, obtained whenever $\left|\Delta \Phi_{0}\right|>\Delta \Phi_{c}$, are reported in Fig. 1(b,d) and Fig. 2(b,c) for input phase $\Delta \Phi_{0}=0.50 \pi$ and $\Delta \Phi_{0}=-0.42 \pi$, respectively. On the contrary, whenever $-\Delta \Phi_{c}<\Delta \Phi_{0}<\Delta \Phi_{\text {c }}$ (cyan domain in Fig. 2(a)), the system follows single loop orbits which correspond to successive unshifted maximally compressed pulse trains, as shown in Figs. 2(d,e) and 2(f,g). Noteworthy, in the case shown in Figs. 2(f,g) which gives the longest measured value of $Z_{1}$, the power initially flows from the sidebands towards the pump, at variance with all the other cases shown in Figs. 1-2, and consistently with the positive sign of $\sin \left(2 \Delta \Phi_{0}\right)$ which determines the initial direction of the power flow $[9,10]$ (see Visualization 1 for all input phase values ranging from $-0.5 \pi$ to $0.5 \pi$ rad. ). From the results reported in Fig. 2, we can conclude that the measured values of the distances for the first two maximal compressions agree well with the estimate from Eq. (3-4) in the observed range (2-6 $\mathrm{km}$ for $Z_{1}$ and 7-9 $\mathrm{km}$ for $Z_{2}$ ). We attribute the slightly larger discrepancy between the numerics and measured data for $Z_{2}$ [magenta dashed line and crosses in Fig. 2(a)] to residual small losses due to imperfect compensation (via the Raman amplification scheme), which have stronger impact on longer distances of propagation. Finally, we point out that, in the experiment, further improving the resolution of the input phase that determines separatrix crossing is extremely challenging due to (i) the intrinsic sensitivity to initial condition and perturbations close to the separatrix; (ii) the need for much longer fibers in turn requiring more involved loss compensation schemes.

In a second set of experiments, we set the initial relative phase to $\Delta \Phi_{0}=0$ and studied the influence of the input sidebands power on the recurrence positions in both the symmetric and asymmetric case. First, we considered symmetric sidebands with

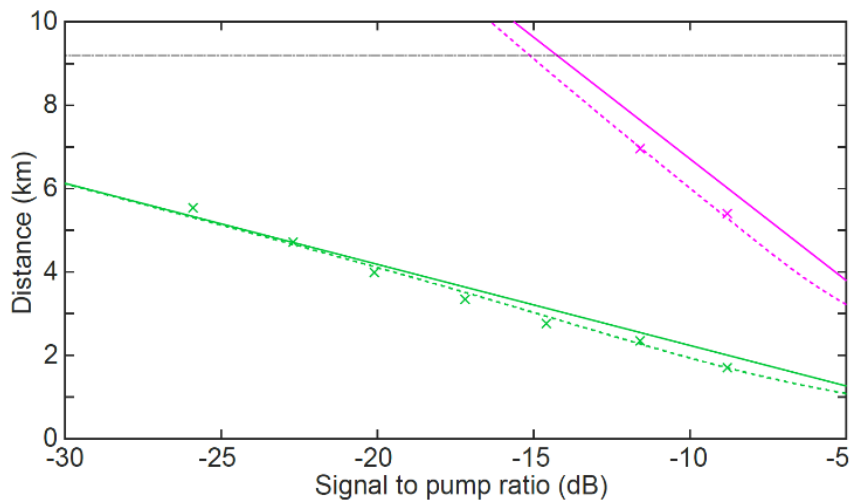

Fig. 3. Position of the first (in green) and second (in magenta) pump minima versus signal (idler) to pump ratio. The crosses correspond to experimental recordings, dashed lines to numerical simulations from NLSE and solid ones to the the theoretical model. The grey line indicates the fiber length. 
a signal/idler to pump ratio varied from $-5 \mathrm{~dB}$ to nearly $-30 \mathrm{~dB}$. The recorded positions of the pump minima are shown in Fig. 3. Crosses account for experiments, solid lines for predictions from Eqs. (3-4) and dashed ones for simulations (green for the first pump minimum and magenta for the second one). The first minimum ranges from about $1 \mathrm{~km}$ to $6 \mathrm{~km}$. We observe a logarithmic dependence of the position on the modulation intensity, as expected from theory [Eq. (3)]. The second pump minimum, however, is expected from Eq. (4) in the range 3 - $18 \mathrm{~km}$ for such variation of the modulation depth. Therefore, only two experimental recordings fall under the length of our $9.2 \mathrm{~km}$ fiber (see the grey line in Fig. 3). They are found in excellent agreement with simulations. From a general point of view, increasing the induced modulation strength clearly shortens the growth and decay cycles. The nonlinear phase acquired by the sidebands through Kerr effect is thus larger and forces the systems to evolve more rapidly toward the critical value of relative phase for which energy exchanges are reversed $[9,10]$.

Second, while maintaining fixed the initial signal to pump ratio at $-10.4 \mathrm{~dB}$ and the initial phase at $\Delta \Phi_{0}=0$, we have decreased the idler power in order to induce a power imbalance between the sidebands and investigate the asymmetric case. Fig. 4 shows the positions of the pump minima when the initial power imbalance between the idler and the signal ranges from $0 \mathrm{~dB}$ to $20 \mathrm{~dB}$. Again, the crosses correspond to experiments, the solid lines to predictions from Eqs. (3-4) and the dashed one to simulations. Here, the distance $Z_{1}$ of the first pump minimum range from about $2 \mathrm{~km}$ to $3 \mathrm{~km}$ and the second one $\left(Z_{2}\right)$ from about $6 \mathrm{~km}$ to $9 \mathrm{~km}$. As shown, the larger the asymmetry the longer it takes to reach the maximum depletion. Interestingly, however, most of the variation occurs while the idler power is still close to the signal one, since the variation rapidly saturate to an asymptotic value for large imbalances. Experimental recordings are in a good agreement with numerical simulations for both minima. Furthermore, in order to illustrate the stability of our measurements we reported the typical error bar for one of the measurements in Fig. 4. The standard deviations for the first and second pump minima are low as they turn out to be about $100 \mathrm{~m}$ and $200 \mathrm{~m}$, respectively. One of the causes of these variations could be the variation of the pump power. Indeed, we checked numerically that varying the pump power by only $2 \%$ give similar deviations.

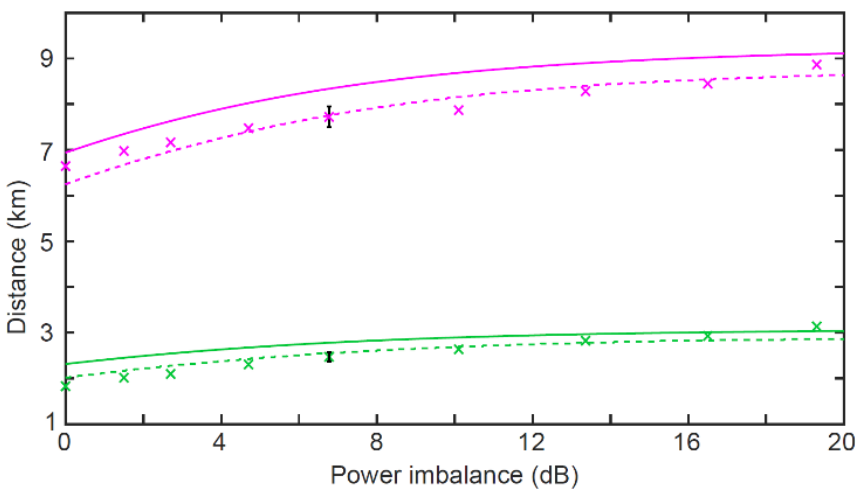

Fig. 4. Position of the first (green) and second (in magenta) pump minima versus power imbalance between signal and idler. The crosses correspond to experimental recordings, dashed lines to numerical simulations from the NLSE and solid lines to theory [Eqs. (3-4)].
In conclusion, we have reported the careful quantitative characterization of the first two induced cycles of conversion and the associated separatrix crossing phenomenon in nonlinear MI. To do that, we used a non-destructive experimental setup enabling to perform distributed measurements of the pump and signal power and phase in a $9.2 \mathrm{~km}$ fiber. We focused on the impact of initial modulation sidebands relative phase and intensity on the positions of the first and second maximally compressed pulse train appearance, comparing with recent analytical estimates by Grinevich and Santini [26]. A good agreement is obtained although the input modulation was relatively strong.

Funding. Agence Nationale de la Recherche (ANR) (Labex CEMPI ANR-11-LABX0007, Equipex FLUX ANR-11-EQPX-0017); European Regional Development Fund (ERDF) (CPER Photonics for Society P4S, HEAFISY); French Ministry for Higher Education and Research (MESR); Hauts de France Regional Council.

\section{References}

1. E. Fermi, J. Pasta and S. Ulam, in Collected Papers of Enrico Fermi Vol. 2 (ed. E. Segré), 977 (Univ. Chicago Press, Chicago, Illinois, 1965).

2. M. Onorato, L. Vozella, D. Proment and Y. V Lvov, Proc. Natl Acad. Sci. USA 112, 4208 (2015).

3. L. Pistone, S. Chibbaro, M. Bustamente, Y. Lvov and M. Onorato, arXiv: 1812.08279

4. T. Tanuiti, H. Washimi, Phys. Rev. Lett. 21, 209 (1968).

5. B. M. Lake, H. C. Yuen, H. Rungaldier and W. E. Ferguson, J. Fluid. Mech 83, 49 (1977)

6. O. Kimmoun, H. C. Hsu, H. Branger, M. S. Li, Y. Y. Chen, C. Khariff, M. Onorato, E. J. R. Kelleher, B. Kibler, N. Akhmediev and A. Chabchoub, Sci. Rep. 6, 28516 (2016).

7. G. Van Simaeys, Ph. Emplit, M. Halterman, Phys. Rev. Lett 87, 033902 (2001).

8. N. Akhmediev, Nature 413, 267 (2001).

9. S. Trillo and S. Wabnitz, Opt. Lett. 16, 986 (1991).

10. G. Cappellini and S. Trillo, JOSA B 8, 824 (1991).

11. M. Conforti, A. Mussot, A. Kudlinski, S. Rota Nodari, G. Dujardin, S. De Bièvre, A. Armaroli and S. Trillo, Phys. Rev. Lett 117, 013901 (2016)

12. A. Bendahmane, A. Mussot, A. Kudlinski, P. Szriftgiser, M. Conforti, S. Wabnitz and S. Trillo, Opt. Express 23, 30861 (2015).

13. A. Mussot, A. Kudlinski, M. Droques, P. Szriftgiser and N. Akhmediev, Phys. Rev. X 4, 011054 (2014).

14. K. Hammani, B. Wetzel, B. Kibler, J. Fatome, C. Finot, G. Millot, N. Akhmediev and J. M. Dudley, Opt. Lett. 36, 2140 (2011).

15. A. Mussot, C. Naveau, M. Conforti, A. Kudlinski, F. Copie, P. Szriftgiser, and S. Trillo, Nat. Phot. 12, 303 (2018).

16. C. Naveau, P. Szriftgiser, A. Kudlinski, M. Conforti, S. Trillo and A. Mussot, Opt. Lett. 44, 763 (2019).

17. D. Pierangeli, M. Flammini, L. Zhang, G. Marcucci, A. J. Agranat, P. G. Grinevich, P. M. Santini, C. Conti and E. DelRe. Phys. Rev. X 8041017 (2018).

18. V. E. Zakharov and L. A. Ostrovsky, Phys. D 238, 540 (2009).

19. N. Akhmediev, A. Ankiewicz, J. M. Soto-Crespo, J. M. Dudley, Phys. Lett. A 375, 775 (2011)

20. J. M. Dudley, F. Dias, M. Erkintalo, \& G. Genty, Nat Phot. 8, 755 (2014).

21. J. M. Dudley, G. Genty, F. Dias, B. Kibler and N. Akhmediev, Opt. Express 17, 21497 (2009).

22. N. Akhmediev and V. I. Korneev, Theor. Math. Phys 691089 (1986).

23. M. Erkintalo, G. Genty, B. Wetzel, J. M. Dudley, Phys. Lett. A 375, 2029 (2011).

24. S. A. Chin, O. A. Ashour, M. R. Belic, Phys. Rev. E 92, 063202 (2015).

25. N. Akhmediev, V.M. Eleonskii, N.E. Kulagin, Theor. Math. Phys. 72, 809 (1987).

26. P. G. Grinevich and P. M Santini, Phys. Lett. A 382, 973 (2018). 


\section{FULL REFERENCES}

1. E. Fermi, J. Pasta and S. Ulam, in Collected Papers of Enrico Fermi Vol. 2 (ed. E. Segré), 977-988 (Univ. Chicago Press, Chicago, Illinois, 1965).

2. M. Onorato, L. Vozella, D. Proment and Y. V Lvov, Route to thermalization in the $\alpha$-Fermi-Pasta-Ulam system, Proc. Natl Acad. Sci. USA 112, 4208-4213 (2015).

3. L. Pistone, S. Chibbaro, M. Bustamente, Y. Lvov and M. Onorato, arXiv: 1812.08279

4. T. Tanuiti, H. Washimi, Self-Trapping and Instability of Hydromagnetic Waves Along the Magnetic Field in a Cold Plasma, Phys. Rev. Lett. 21, 209 (1968).

5. B. M. Lake, H. C. Yuen, H. Rungaldier and W. E. Ferguson, Nonlinear deep water waves: theory and experiment. Part 2. Evolution of a continuous wave train, J. Fluid. Mech 83, 49 (1977).

6. O. Kimmoun, H. C. Hsu, H. Branger, M. S. Li, Y. Y. Chen, C. Khariff, M. Onorato, E. J. R. Kelleher, B. Kibler, N. Akhmediev and A. Chabchoub, Sci. Rep. 6, 28516 (2016).

7. G. Van Simaeys, Ph. Emplit, M. Halterman, Experimental Demonstration of the Fermi-Pasta-Ulam Recurrence in a Modulationally Unstable Optical Wave, Physical Review Letters 87, 033902 (2001).

8. N. Akhmediev, Nonlinear physics: déjà vu in optics, Nature 413, 267268 (2001).

9. S. Trillo and S. Wabnitz, Dynamics of the modulation instability in optical fibers, Opt. Lett. 16, 986-988 (1991).

10. G. Cappellini and S. Trillo, Third-order three-wave mixing in single-mode fibers: exact solutions and spatial instability effects, JOSA B 8, 824-838 (1991).

11. M. Conforti, A. Mussot, A. Kudlinski, S. Rota Nodari, G. Dujardin, S. De Biévre, A. Armaroli and S. Trillo, Heteroclinic structure of parametric resonance in the nonlinear Schrödinger equation, Phys. Rev. Lett 117, 013901 (2016)

12. A. Bendahmane, A. Mussot, A. Kudlinski, P. Szriftgiser, M. Confori, S. Wabnitz and S. Trillo, Optimal frequency conversion in the nonlinear stage of modulation instability, Opt. Express 23, 30861-30871 (2015).

13. A. Mussot, A. Kudlinski, M. Droques, P. Szriftgiser and N. Akhmediev, Fermi-Pasta-Ulam recurrence in nonlinear fiber optics: the role of reversible and irreversible losses, Phys. Rev. X 4, 011054 (2014).

14. K. Hammani, B. Wetzel, B. Kibler, J. Fatome, C. Finot, G. Millot, N. Akhmediev and J. M. Dudley, Spectral dynamics of modulation instability using Akhmediev breather theory, Opt. Lett. 36, 2140 -2142 (2011).
15. A. Mussot, C. Naveau, M. Conforti, A. Kudlinski, F. Copie, P. Szriftgiser, and S. Trillo, Fibre multi-wave mixing combs reveal the broken symmetry of Fermi-Pasta-Ulam recurrence, Nature Photonics 12, 303-308 (2018).

16. C. Naveau, P. Szriftgiser, A. Kudlinski, M. Conforti, S. Trillo and A. Mussot, Full-field characterization of breather dynamics over the whole length of an optical fiber, Opt. Lett. 44, 763-766 (2019).

17. D. Pierangeli, M. Flammini, L. Zhang, G. Marcucci, A. J. Agranat, P. G. Grinevich, P. M. Santini, C. Conti and E. DelRe, Observation of FermiPasta-Ulam-Tsingou recurrence and its exact dynamics, Phys. Rev. X 8, 041017 (2018).

18. V. E. Zakharov and L. A. Ostrovsky, Modulation instability: the beginning, Phys. D 238, 540-548 (2009).

19. N. Akhmediev, A. Ankiewicz, J. M. Soto-Crespo, J. M. Dudley, Universal triangular spectra in parametrically-driven systems, Phys. Lett. A 375, 775-779 (2011).

20. J. M. Dudley, F. Dias, M. Erkintalo, \& G. Genty, Instabilities, breathers and rogue waves in optics, Nature Photonics 8, 755-764 (2014).

21. J. M. Dudley, G. Genty, F. Dias, B. Kibler and N. Akhmediev, Modulation instability, Akhmediev and continuous wave supercontinuum generation, breathers Opt. Express 17, 21497-21508 (2009).

22. N. Akhmediev and V. I. Korneev, Modulation instability and periodic solutions of the nonlinear Schrödinger equation, Theor. Math. Phys 69 1089-1093 (1986).

23. M. Erkintalo, G. Genty, B. Wetzel, J. M. Dudley, Akhmediev breather evolution in optical fiber for realistic initial conditions, Phys. Lett. A 375, 2029-2034 (2011).

24. S. A. Chin, O. A. Ashour, M. R. Belic, Anatomy of the Akhmediev breather: cascading instability , first formation time, and Fermi-PastaUlam recurrence. Phys. Rev. E 92, 063202 (2015).

25. N. Akhmediev, V.M. Eleonskii, N.E. Kulagin, Theor. Exact firstorder solutions of the nonlinear Schrödi,ger equation; Math. Phys 72 809-818 (1987).

26. P. G. Grinevich and P. M Santini, The exact rogue wave recurrence in the NLS periodic setting via matched asymptotic expansions, for 1 and 2 unstable modes, Phys. Lett. A 382, 973-979 (20 
